\title{
Content-Based Image Retrieval Using Texture Color Shape and Region
}

\author{
Syed Hamad Shirazi \\ Department of IT ,Hazara University \\ Mansehra, Pakistan \\ Arif Iqbal Umar \\ Department of IT, Hazara University \\ Mansehra, Pakistan
}

Saeeda Naz

Department of IT, Hazara University, Mansehra

GGPGC No.1, Abbottabad, HED, KPK, Pakistan

\begin{abstract}
Interests to accurately retrieve required images from databases of digital images are growing day by day. Images are represented by certain features to facilitate accurate retrieval of the required images. These features include Texture, Color, Shape and Region. It is a hot research area and researchers have developed many techniques to use these feature for accurate retrieval of required images from the databases. In this paper we present a literature survey of the Content Based Image Retrieval (CBIR) techniques based on Texture, Color, Shape and Region. We also review some of the state of the art tools developed for CBIR.
\end{abstract}

Keywords-CBIR; Color Space; Relevance Feedback; Texture Features; Shape; Color

\section{INTRODUCTION}

Content based image retrieval (CBIR) has been an active research area since 1970 . It applications has increased many fold with availability of low price disk storages and high speeds processors. Image databases containing millions of images are now cost effective to create and maintain. Image databases have significant uses in many fields including medicines, biometric security and satellite image processing. Accurate image retrieval is a key requirement for these domains. Researchers have developed several techniques for processing of images databases [1]. These include techniques for; sorting, searching, browsing and retrieval of images. Traditional image retrieval approach interprets image by text and then use textual information to retrieve images from textbased database management system. This method has several drawbacks; it uses keywords associated with images to retrieve visual information. It is very tedious and time consuming. It is hard to describe the contents of different types of images with textual representation. Keywords due to their subjective natures fail to bridge the semantic gap between the retrieval system and the user demands; consequently the accuracy of the retrieval system is questioned. The keyword for describing images becomes inadequate in large databases. It is not scalable.

\author{
Noor ul Amin Khan \\ Department of IT, Hazara University \\ Mansehra, Pakistan
}

Muhammad Imran Razzak

King Saud bin Abdulaziz University for Health Sciences, Riyadh, Saudi Arabia

\author{
Bandar AlHaqbani \\ Technology Control Company, \\ Riyadh, Saudi Arabia
}

Content Based Image Retrieval (CBIR) is a powerful tool. It uses the visual cues to search images databases and retrieve the required images. It uses several approaches and techniques for this purpose. The visual contents of images, such as color [2], texture [3]-[5], shape [6] and region [7], are extensively explored for indexing and representation of the image contents. These low level features of an image are directly related to the contents of the image. These image contents could be extracted from image and could be used for measuring the similarity amid the queried image and images in the database using different statistical methods. In content-based retrieval systems different features of an image query are exploited to search for analogous images features in the database [8]-[10].

Various techniques based on texture features have been proposed in the literature. These include both statistical approaches and spectral approaches. Mostly these techniques are not able to capture accurate information. Color is most reliable feature which is easier to implement for retrieval of image. Color is easier to implement because it is robust to background compilation. It is free of image size and its orientation. The most common approach for color features extraction of images is histogram. Color histogram illustrates the color distribution in image and it entails low computational cost. Color is also insensitive to trivial deviations in the assembly of image. The main shortcoming of color histogram is that they cannot fully consider spatial information and they are not exclusive [11].

Different images having same color distribution yield almost similar histograms. Besides, in diverse lighting conditions analogous images having same point of view generate dissimilar histograms. Despite of using the information extracted from image, most of the CBIR systems yield imprecise outcomes. Because it is challenging to relate the low-level features with the high-level user semantics. This problem is known as semantic gap [12]. To over-come the problem of semantic gap, relevance feedback methods are used in [12], [13]. Relevance feedback method provides a 
mechanism for CBIR system to allow the system to learn about the features best serve the user's interests. This method enable user to assess the images retrieved by the current query and assign them values which indicates their relevance.

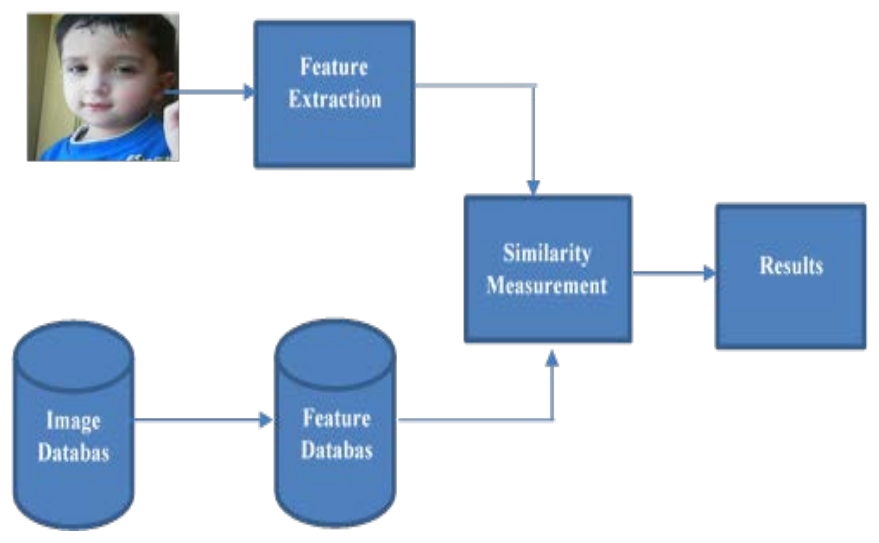

Fig. 1. CBIR system overview

\section{RELATED WORK}

Research on CBIR could be bifurcated into two groups on the basis of the features used to retrieve the required image. Early approaches used a single feature out of the available features namely shape, texture, color and region for retrieval of the required image. Results of single feature based retrieval systems were not satisfactory because generally image contains several visual features. The current approaches use different combination of the visual features to retrieve the required image [1], [5], [14], [15].

The shape descriptor also provides dominant information in image retrieval because shape is the only source through which humans can recognize objects. The shape feature can be retrieved by two methods boundary based shape feature extraction and region based shape extraction. The boundary based technique is based on outer boundary while the region based technique is depending on the whole region [16].

An efficient CBIR system with better performance is presented by using the wavelets decomposition of image; they have generated the composite sub-band gradient and the energy distribution pattern string from the sub images of are generated by means of wavelet decomposition to the input image [1]. For filtering out the undesired images a technique based on energy distribution pattern strings fuzzy matching is used. The resultant images are compared with query image after filtering. The system is tested on the database of 2400 images.

Texture has no formal definition but intuitively it provides measure of properties such as coarseness, regularity and smoothness. It plays a role in human visual perception and interpretation. Texture describes in three approaches, namely, structural, statistical and spectral. In structural approach the texture is formed of small texture elements called 'texels' by following placement rules. The statistical method assumes texture by means of statistical grey level features of image pixels. The spectral approach is based on filtering theory in frequency domain and power density function. The structural approach is not so prevalent because majority of the natural structures contain asymmetrical shapes [3]. In CBIR the conventional Gabor representation and the features extracted by it has demonstrated poor performance especially in retrieval of the rotated and scaled texture images. Most of the existing retrieval systems rely on several stages of transformations for making scaling and orientation at the cost of too much computational complexity and degradation in performance.

In [5] experimentations on large data set of textured images are performed. The authors have shown that the performance and efficiency of Gabor wavelet performs better as compared to the conventional orthogonal wavelet based features. The conventional Gabor transform have some limitations. The orthogonal based sets are not formed by Gabor function that is why its representation is not solid and it consumes more computation cycles and more memory. In [17] a CBIR system is presented. It extract textural and color features of image. To extract color features color histogram is used. Gabor filter is used for the extraction of texture features. The image phenomenon of image retrieval is carried out on the basis of linear combination using texture, structural and color histogram values. Image contents are retrieved on the basis of three features and a selection technique for feature extraction is also used to select optimal features for the enhancement of the detection rate and to minimize the computational complexity of the image retrieval.

A color distribution based system is proposed in [2]. It computes the probability of the incidence of identical pixel colors for each pixel and its neighbor pixels in the image. The difference among pixels is calculated and applied to the entire image. All pixels in the image are grouped by using K-means Clustering algorithm. The technique is applied to 3 databases and better results are reported. The technique is not suffering from image displacement and rotation. Analyzing images in real-time dynamic environment is not feasible. A system is proposed in [18] that analyze images in real-time environment. It extracts shape and color to represents image contents. The system uses C-Means clustering algorithm to segment the images and extract its boundaries. Fast Fourier Transform is applied to provide an array of vector corresponding to a region. For color features HSI color model is used. Similarity Matching Algorithm using distance measures among the feature vector is applied to match the queried images.

The capability of a CBIR approaches is fully dependent on the features retrieved from the image. Frequently it is observed that there is a semantic gap between the visual features and semantic content of an image. The semantic gap could be decreased by extraction of more effective features. This is a challenging area in CBIR research. To overcome the semantic gap different machine learning techniques are used. In [19] and [20] SVM is used to extract the image features accurately and retrieve the desired image efficiently. In [21] hierarchical methodology is used to retrieve an iris image is presented. This technique is based on an innovative indexing method for an iris database. Two distinct features are exploited to extract the contents of iris image i.e. iris color is used to form the basis for the indexing of image while texture of image is used to retrieve image from the indexed iris database. The undesired images are filtered out by using the color feature; the images having no similarity with the query image color are filtered out. The proposed technique is evaluated over noisy iris images. It 
produced better results. A novel image retrieval scheme (ICTEDCT-CBIR) based on curvelet transform is presented in [11], this model integrates Curvelet multi-scale ridglets with region-based vector codebook sub band clustering to extract dominant color feature and texture analysis. The advantage of Multi-scales of Curvelet transform is that it restores sparsity by curtailing the redundancy across multi-scales. The Curvelet based technique is better than the previously discussed techniques because along with Gabor filter, the wavelets are mostly used to retrieve image contents. The wavelets perform well while presenting point singularities but it ignores the geometric properties of shapes. It also ignores the use the symmetry of image edges.

Curvelet based technique is proposed in [22]. It uses Curvelet transform along with vocabulary tree for feature extraction and image retrieval. The Gabor wavelet transform is used in conjunction with Curvelet transform. For feature vector generation Histogram with vocabulary tree is used. The proposed algorithm is compared with Gabor transform and wavelet transform. The proposed algorithm showed better results because of capturing more accurate edge information.

In [6] a new approach based on three popular algorithms that are: color histogram, texture and moments invariants is proposed. The three algorithms are used to ensure capturing of the regularity of image edges. They have extracted the texture features by using Gabor filter. Shape features of an image are retrieved by using the moment invariants and the color features of the image. The proposed method is evaluated by using the typical precision and recall measure. The main drawback of surveillance images is that there quality is low. That makes the detection, recognition and retrieval process difficult. Environmental factors and use of low resolution cameras are the main reasons of low quality of surveillance images. Fundamental contributors in environmental factors are fog, rainfall and snow which can affect the quality of image. These factors affect the image details very badly and generate noise that makes the detection of objects in image and retrieval of image information more difficult. On the basis most significant features selection a CBIR model is presented in [23]. In this model Gabor filter and 3D histogram are combined together for feature extraction. Gabor filter is used to extract texture feature while 3D histogram is used for color feature extraction. They have used Genetic algorithm to get finest boundaries of the intervals. The model has reduced the retrieval time by introducing new method for feature selection. Because the traditional methods are based on the curse of dimensionality this causes the degradation in performance and increases computations. The effectiveness of CBIR depends on image descriptors that are being used to retrieve contents of image [24] like distance functions, color descriptor, texture descriptor and shape descriptor.

In [25] a new approach to extract color and texture features of image for CBIR is proposed. They identified the low level features of color and texture for CBIR by using two color histogram function and their comparison. it combined texture, color and shape descriptors for image retrieval. The paper focuses on feature extraction and representation. In [26] an image retrieval approach based on fuzzy KNN classifier is proposed. It allocates an initial semantic label to database images. In this method the assigned labels to the images are modified steadily by relevance feedback. Several measures based on similarity were observed for three kinds of visual features. Genetic algorithm was used to assign optimum weights for each type of feature and to find out their components. For the residual 800 images of the image database, the proposed approached assigned outputs classifier as initial weights to the associated links of the network. During the image contents retrieval session, the network weights are turned in to the relevance feedback from the users.

The semantic gap between low level visual features and high level semantics is reduced by means of instantaneous feature selection and variations [27]. They used orthogonal wavelets for texture retrieval and color histogram to extract the color feature. The proposed method is applied on 1000 images database and the method produced better results. Texture, color and shape descriptor are used for image retrieval in [28]. The image is determined by utilizing color quantization algorithm along with the cluster merging technique. The approach retrieves texture by using steerable filter decomposition and achieved the shape descriptor by using Zernike moments of image moments. Zernike moment is more robust to noise.

In [29] Neural networks are exploited for CBIR. The images for classification are divided into foreground images and background images. Region based segmentation technique is used to get the region of the objects. Texture feature are extracted by using wavelet transform to retrieve shape based. They used neural network for back propagation learning.

In [30] a new descriptor for the retrieval of image is called micro-structure descriptor (MSD) is proposed. The MSD is defined on the basis of edge orientation similarity. This technique based on color, it extracts features and effectively combines them with shape, color, texture and color layout features entirely for image retrieval. The proposed method is verified on the database of 15000 images. The results are compared with Gabor features and multi-textons histograms. It produced better results. Feature selection is a common problem in CBIR. Image characterization with fewer numbers of features decreases the computational cost. Edge is also a robust feature for image characterization. A robust method for edge detection and extraction is proposed in [31]. They computed global features by means of using gray level and the shape information. This method does not require preprocessing and image segmentation. A CBIR System which first performs image segmentation by dividing image into various regions is proposed in [32]. The regions are used to retrieve image. The image searching is performed on the basis of image regions that have similar association with the regions that exists in image query. For image retrieval color and texture features are used in [32-33]. An image retrieval system based on the idea of motif co-occurrence matrix is presented (MCCM) in [34]. This technique differentiates pixels and then converts them into basic graphic. Probability of occurrence of the neighboring areas in the image is calculated to acquire the color variance concerning the adjacent pixels.

In [2] a novel CBIR system based on three methods: Initially co-occurrence matrix (CCM) is computed for color feature. The CCM matrix is used to analyze probability of 
occurrence of pixels having same color and the adjacent pixels in the image. Second the difference between pixels of scan patterns (BDPSP) is computed to find out the variance among all pixels of scan patterns. The third method is color distribution for K-mean algorithm. It is based on color histogram in which each color pixel is substituted by any color that is utmost related to the existing color. The K-mean algorithm divides all the pixels into k clusters. A CBIR system based on three algorithms viz. feature extraction, image mining and the rule based is proposed in [35]. The first algorithm globally extracted the color and texture features from the image. It is considered that these features are invariable to the image transform and could be used for the detection of the objects. The second algorithm uses the image mining method that implies clustering algorithm to retrieve hidden knowledge from the image. The third algorithm uses the rules based on relevance feedback to filter the results and to improve the clusters.

\section{A. Texture}

Texture is mostly used in CBIR. Texture classification and texture based image segmentation are the challenging tasks [1], [3] [4] [5]. Texture is based on neighborhood. The texture feature provides vital information for the classification of image because it is useful in describing the contents of numerous real world images such as fruit, skin, bricks, trees, clouds in the sky and fabrics. Texture helps in describing the high level semantics for image retrieval. The main problem in texture retrieval is the scale selection; most of the literature work has neglected the importance of scale selection for the computation of texture. Statistical methods are used for the analysis of image grey level spatial distribution. The probability of the co-occurrence of gray values in distinct orientations and at different distances is calculated by these methods. Histogram is used for statistical information generation. The co-occurrence matrix is used in [2], [34] for texture identification. Texture is examined by means of texture primitives for this purpose Geometrical approach is adopted. This analysis is performed by taking the geometrical characteristics of the primitives, such as: size, shape, area, and length. In [34] [40] grids are extracted from related vectors which join the primitives' centroid.

This type of analysis is challenging for various natural textures, due to the presence of the irregularity in primitives. For example, it is to describe a wall of bricks by means of brick primitive and placement rule for this can be very simple as compared to describe the clouds in sky. Because clouds have a lot of variations in shape, size and position. Model based approaches depend on the assembly of image models. These models can be used to define and produce textures. In several systems, the image texture feature is extracted on basis of the pixels texture property or small blocks present in a small region [49]. They have calculated the mean values of the texture of all the $4 * 4$ blocks and used it as a region feature.

Such features suffer from problems because they cannot define the texture property of the whole region. Image retrieval system proposed in [50] relies on texture features only. The texture feature similarity is extracted by using wavelets and gradient vector decomposition. The system used two characteristics of each image namely the image detail features and the coarse features. The coarse feature is used to discard the undesired images and the detail features of image are used for the related image retrieval.

\section{B. Color}

Color points create color space. Various color spaces based on the perceptual concepts are used for color illustration. There is no universally accepted criterion to judge color space. The desire features of a color space are its completeness, compactness, uniformity, and user orientedness. Completeness shows that it must comprise of all the perceptible colors. Uniformity indicates the closeness among the different colors. It is directly connected with the psychological association among the colors. Various color description are used in [36]. The color spaces are more related to human perception and widely used in CBIR. That is comprised of RGB, LUV, HSV, YCrCb and LAB [37]-[39].

Color plays vital role in extraction of information from images. Color histograms are frequently used in CBIR systems [6], [17], [22], [40]. Histogram is useful though its global characterization is weak. Histograms are not efficient in handling noise because they are very sparse. In order to overcome limitations of the histogram other color features such as color moments and color sets representations are applied for image retrieval [6]. The color feature selection depends on the result of image segmentation. If the segmentation provides objects having heterogeneous color, then average color will not be a good option for image retrieval. Most of the CBIR systems function on the color images that are not preprocessed. The image capturing devices introduced noise in the images. If these color images are preprocessed then image retrieval accuracy of CBIR system will be enhanced. Noise from color images could be removed by filters. Several noise removing filters are proposed in the literature [41-43].

Literature survey shows three methods for color analysis. The Global approach focus on the image color information globally without applying any segmentation or preprocessing technique during feature extraction of the image. This approach is efficient but do not provide any information associated to the spatial distribution of colors. It generates color histogram as feature vector [44], [45]. Equal size regions approach decomposes the image into small portions of fixed size on the basis of the small regions separately. This approach provides more spatial information. It uses local color histogram to generate feature vectors [6], [46]. The third approach is the segmentation based approach that divides the image into different regions of different sizes. Clustering algorithm is responsible for the division that causes an additional complexity to feature extraction process [47], [48].

\section{Shape and region}

Shape can be defined as the feature surface configuration of an outline, object or contour. The shape feature is used to separate objects from the background and surrounding by its outline representation. It can be categorized into two broad categories. Region based and boundary based image retrieval. Regional properties of images can be utilized, and segmentation can be used for color, shape feature extraction and spatial position of the regions. In [7] a technique based on region is used for image retrieval. They used image 
segmentation in small regions. Local properties of different regions are helpful in matching objects of the images. This makes CBIR effective. An approach based on region matching, that uses combination of different features like location, color, and shape is developed within the MPEG-7 framework. They have used these integrated features (shape, location and color) for the indexing of significant regions within each image. It also indexes various arrangements of regions. Similar images are clustered and Hash structure is used to capture resulted indices and Meta data. The images are retrieved on the basis of color, shape or location and their combination. Results indicate that CBIR can be made more effective and efficient by using segmentation to retrieve the images. In [51] specific regions of the objects of most interest are computed by using low level features.

The results indicate that the simple thresh hold technique is more accurate and produce better results than the methods which are more complex and based on statistics. There are three methods for the detection of salient objects in an image; the non-parametric measure [52], the thresh hold based measure in which threshold is manually selected and the modified Hubert index [53]. For the classification of objects in images, image can be divided into foreground and background regions [54]. Objects regions are extracted by using region segmentation techniques. Shape-based texture features are mined from images by using wavelets transform. The method is used to automatically classify objects of images. Neural networks are also used for the classification of objects. The selective visual attention can play a key role in human understanding of image by intuitively focusing on some salient parts. The interest of CBIR systems users is confined to selective portions of image and the rest of image is irrelevant.
A selective visual attention model is proposed in [55]. In most of the region based approaches, the shape descriptors use the pixel information with in the region of the shape. Such techniques are applied for general applications. The region based techniques use moment descriptors to define shape [56], [57]. These moment descriptors are consisting of Legendre Moments, Geometric Moments, Zernike Moments and PseudoZernike Moments. The region based shape descriptors are retrieved by means of spatial domain and most of them are suffering from noise and shape variations. Generic Fourier descriptor is applied to general applications. The Generic Fourier descriptor is extracted by using spectral domain. Shape finer features in both circular and radial directions are captured by using 2-D Fourier transform [58]. A region based retrieval method is presented in [59]. This method combines motif cooccurrence with spatial relationships. Local information is extracted by Local space filling curves to calculate motif cooccurrence matrix for texture description. It decomposes each image into coherent segments.

The motif co-occurrence matrix is computed for all regions. Spatial relationships between these regions are calculated. During the process of image retrieval each of the regions is allocated its own MCM and it is used as a feature vector. It is used for satellite images.

Semantic based CBIR has become very popular and attracted interest in current decade. In [60] [61] region based image retrieval system with high level semantics learning is presented. This method is more flexible because supports both query by region of interest and query by keyword. It divides image into diverse regions and for each region low level features are extracted. Decision tree based learning method (DT-ST) is used to achieve high level concepts.

TABLE I. Color, TeXture AND Shape FeAtures UsEd In CBIR Systems

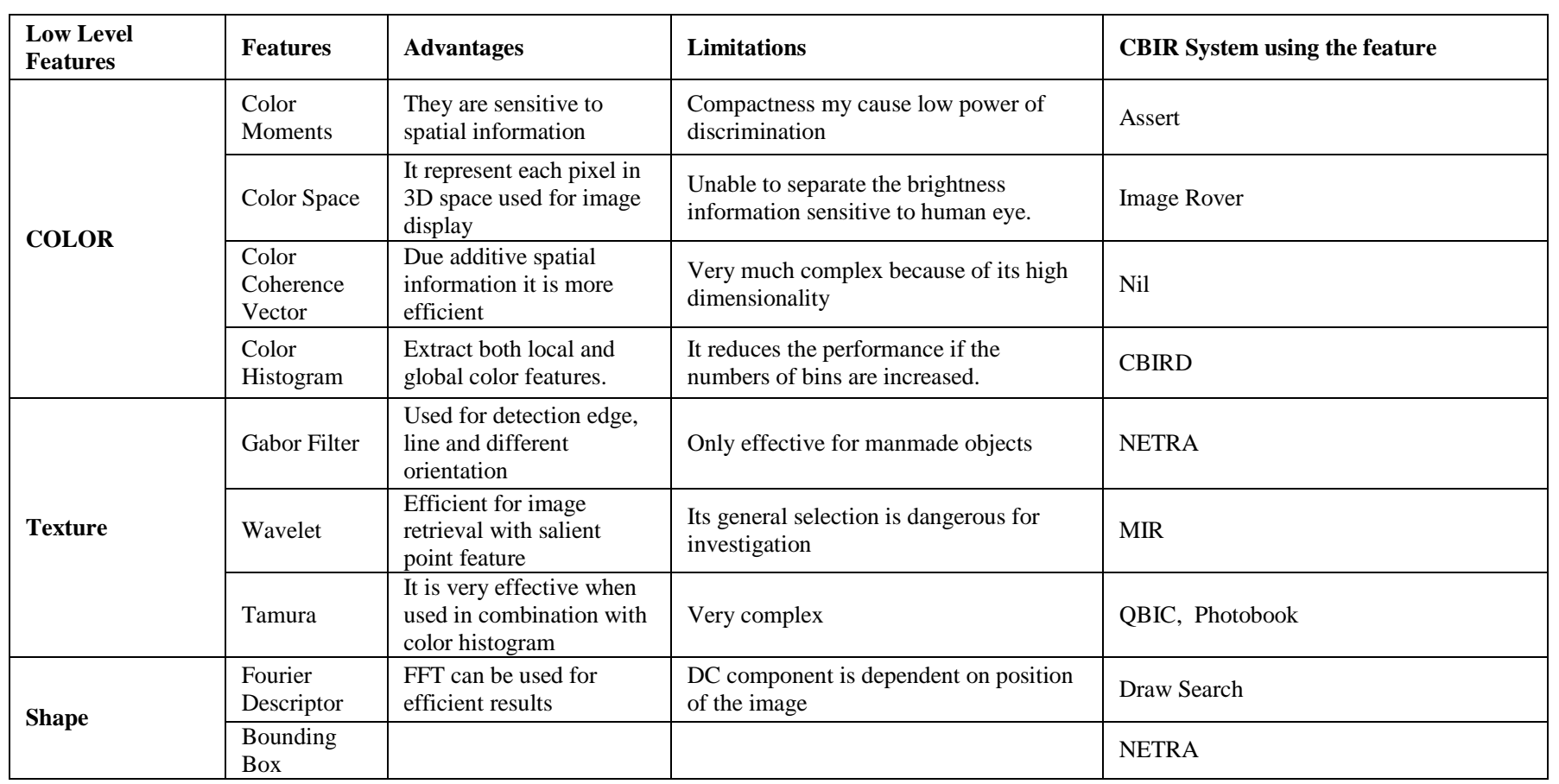


A set of images whose concept matches with the query image is retrieved. This approach has significantly enhanced the image retrieval process as compared to the conventional CBIR systems. The proposed DT-ST decision tree induction method form image semantics learning is better because it uses the semantic template to discretize continuous valued regions feature. This algorithm has improved classification performance of the tree and it has outclassed the wellorganized ID3 and C4.5 algorithms

\section{SIMILARITY MEASURES}

An image may contain visual information or semantic information. The visual information can be represented in form of shape, color, texture and spatial relations. The extracted visual features are considered as feature vector that are kept in feature database. The retrieval process is performed by different similarity measuring techniques that match the resemblance between the query image and the stored image in the database. The result of the query may not be a single image. It is a series of images categorized by the similarity of retrieved image with the query image. Retrieval performance of the CBIR systems is affected by similarity measures. Similarity measures are used to find the correspondence of the query image with the image stored in the database. This section contains some similarity measures that are used in the literature. We denote $\mathrm{I}=$ Query image $\mathrm{J}=\mathrm{Image}$ in database $\mathrm{fi}$ (I) $=$ represent the number of pixels in, $\mathrm{i}^{\text {th }}$ bin of query image.

\section{A. Minkowski-Form Distance}

The Minkowski-form distance La is apt for the computation of distance between two images when each of the image feature vectors having equal importance and are independent from each other. It is defined as:

$$
D(I, J)=\sqrt{\sum_{i=1}|f(i)-f(i)|^{a}}
$$

If $a=1,2 \ldots . \operatorname{Ln} \mathrm{D}(\mathrm{I}, \mathrm{J})$ is $\mathrm{L} 1, \mathrm{~L} 2 \ldots . . \mathrm{Ln}$ then this is called Euclidean distance. The minkowski-form distance for color and shape feature is used in Netra [62].

\section{B. Histogram Intersection}

Histogram intersection is the special case of Euclidian distance. Many CBIR systems used it for computation of the similarity among color images. Histogram intersection of image I and image $\mathrm{J}$ can be measured as:

$$
S(I, J) \frac{\sum_{i=1}^{N} \operatorname{Min}((f i(I), f i(J))}{\sum_{i=1}^{N} f i(J)}
$$

It has been revealed that histogram intersection is reasonably tactless to variations in image resolution, histogram size, occlusion, depth, and viewing point.

\section{Quadratic Form Distance}

The problem in Minkowski distance is that it treats all the bins independently. It ignores the similarity between certain pairs of bins having same features. This problem is addressed by Quadratic Form Distance.

$$
D(I, J)=\sqrt{\left(\left(F(I)-F(J)^{T} A F(I)-F(J)\right.\right.}
$$

Where $A=\left[a_{i j}\right]$ denote the similarity among $\mathrm{i}$ and $\mathrm{j}$ bins. Vectors $\mathrm{Fi}$ and $\mathrm{Fj}$ enlist each of the items in $\mathrm{fi}(\mathrm{I})$ and $\mathrm{fi}(\mathrm{J})$. Many CBIR systems use quadratic form distance [63]. It can produce better retrieval results as compared to histogram intersection and Euclidean distance methods.

\section{Mahalanobis Distance}

Another metric suitable for all dimensions of image feature vector is defined as.

$$
D(I, J)=\sqrt{(F I-F J)^{T} C(-1)(F I F J)}
$$

The $C$ represents co variance matrix of the feature vectors. In case the feature dimensions are independent it will become so simple. In such type of situation we need only variance of each feature component.

\section{RELEVANCE FEEDBACK IN CBIR}

Currently in CBIR systems an effective tool relevance feedback is used to bridge the semantic gap and improve the retrieval rate. It is the supervised learning approach. The focus of this approach is on interaction between user and the search engine. The user is responsible for the labeling of semantically positive or negative feedbacks.

There are three categories of relevance feedback techniques namely; query point movement method [64], Reweighting method [65] and Expectation Maximization method [66]. The query point movement technique focus on the improvement of the approximation of the ideal query point. It diverts it to the positive examples and far from the negative examples. In reweighing method the weight of each feature element is modified. Most of the work done in this area has used SVM (Support Vector Machine) for the pattern classification. it is a small and simple learning method [67]. SVM is considered as a strong candidate for classification because of several reasons: its generalization ability, fast learning, flexibility and assessment of relevance feedback.

Active SVM learning method used in [65] split the image space. In this method the positive samples are considered furthermost from the boundary of classifier on the positive side. The most informative samples are adjacent to the boundary for user labeling. Relevance feedback is an approach that reflects human interaction to rectify high level concepts defined by low level features. It is basically used in text based retrieval [8]. In the application of using relevance feedback first query image is selected then user marks the relevant images from retrieved result and modifies the query.

In [68] the authors automatically refined high level concepts on the basis of relevance feedback from the user. SVM is utilized to create a classifier with minimized Vapnik Chervonenkis (VC) dimension [69] based on structural risk minimization principle. The work on CBIR systems are widely discovered in the past decade [70]. The semantic gap existing between the high level and low level concepts is handled with Relevance feedback technique [71]. SVM has been considered as the most powerful tool to enhance the retrieval rate of image retrieval systems. SVM is considered is also considered as a leading technique for classification in relevance feedback systems [72-73]. The learning problem in SVM-based relevance feedback approaches is considered as a strict binary classification task. In real world relevance feedback applications this assumption is incorrect. 
TABLE II. CBIR SYSTEMS AND QUALITATIVE COMPARISON

\begin{tabular}{l}
\hline \multicolumn{5}{|c|}{ Texture } \\
\hline
\end{tabular}

The systems based on color feature usually retrieve images having similar color. The problem in texture based retrieval is that they produce in-accurate retrieval results. It does not always produce images that have noticeably the same texture, the texture may be effective only when the database comprises of many images with a prevailing texture. Searching for shape feature yields frequently best outcomes. Superficially for matching purpose shape features are not the best ones. Indexing data structures are not applicable for small databases. Linear search is the better alternative of indexing structure for such kind of databases. Simple matching of hundreds of images in efficient manner is not a problem for modern computers In terms of efficiency, effectiveness, and flexibility, it is very hard to decide that how effective are the CBIR systems in terms of precision, accuracy and recall. Accuracy is defined as the ratio of appropriate images to the total retrieved images from database and recall is defined as the percentage of appropriate images to all relevant images. Numerous articles about CBIR systems contain figures showing the precision and recall. Most of these results are decent, but it is difficult to validate them. It is extensively accepted that most current CBIR systems are using the low level image features (color, texture, shape) for image retrieval. The high level semantics will the main driver of the future CBIR systems. One way to attain this is to make the systems capable of identifying objects and scenes. This is a challenging task at implementation level but it should be practicable for applications used in specific domains [74].

\section{CONCLUSION}

The content based image retrieval techniques are still under research. Various algorithms are proposed to improve the image retrieval capability of CBIR systems by using content based approaches. Majority of these approaches are based on a single algorithm and ignore the existence of others. Approaches which are relying on a single specific algorithm like color, texture or shape, such type of approaches can work successfully on specific images but when varied types of images are taken into account their performance is degraded.

In this paper we have presented different techniques used for image retrieval. Some of these approaches were single feature based and some of them were the combinations of these features (Texture, Color, Shape and region). Most of the studied techniques lack accuracy and are unable to overcome 
the semantic gap between the user and the CBIR system. This area of CBIR systems is a hot research area with huge potential for research and development of better image retrieval techniques.

\section{REFERENCES}

[1] S. D. P.W. Huang, "Image retrieval by texture similarity," Pattern Recognition, vol. 36, p. 665- 679, 2003.

[2] Y. C. C.H. Lin, R.T. Chen, "A smart content-based image retrieval system based on color and texture feature,” Image Vis. Comput., vol.27 (6), p. 658-665., 2009.

[3] A.-W. R.C. Gonzalez, R.E. Woods, Digital Image Processing. Reading, MA, 1992.

[4] K.-K. M. Ju Han, "Rotation-invariant and scale-invariant gabor features for texture image retrieval," Image and Vision Computing, vol. 25, p.14741481, 2007.

[5] B. C. M. Kokare, P.K. Biswas, "Texture image retrieval using rotated wavelet filters," Pattern Recognition Letters, vol. 28, p. 1240-1249, 2007.

[6] A. J. K. Iqbal, M. O. Odetayo, "Content-based image retrieval approach for biometric security using colour, texture and shape features controlledby fuzzy heuristics," Journal of Computer and System Sciences, vol. 78,p. 12581277, 2012.

[7] X. L. M.A. Nascimento, V. Sridhar, "Effective and efficient regionbased image retrieval," Journal of Visual Languages and Computing, vol. 14,p. 151-179, 2003.

[8] W. Grosky, "Image retrieval-existing techniques, content-based (cbir) systems," Department of Computer and Information Science, University of Michigan-Dearborn, Dearborn, MI, USA, vol. 14, 2010.

[9] X. Q. M. Royal, R. Chang, "Learning from relevance feedback sessions using a k-nearest-neighbor-based semantic epository,” IEEE International Conference on Multimedia and Expo (ICME07), Beijing, China,pp. 1994-1997, 2007.

[10] X. Q. K. Shkurko, "A radial basis function and semantic learning space based composite learning approach to image retrieval,,” IEEE International Conference on Acoustics, Speech, and Signal Processing(ICASSP07), vol. 1, p. 945-948., 2007.

[11] S. M. Youssef, "Ictedct-cbir: Integrating curvelet transform with enhanced dominant colors extraction and texture analysis for efficient content-based image retrieval," Computers and Electrical Engineering,vol. 38, p. 13581376, 2012.

[12] S. S. A. G. A.W.Smeulders, M. Worring and R. Jain., "Content-based image retrievalbat the end of the early years," IEEE Transactions on Pattern Analysis and Machine Intelligence, vol. 22, pp. 13491380,2000 .

[13] A. T. P. Bugatti and C. Traina., "Improving content-based retrieval of medical images through dynamic distance on relevance feedback,"In Proceedings 24th International Symposium on Computer-Based Medical Systems(CBMS), pp. 1-6, 2011.

[14] Y. Chun., "Content-based image retrieval using multiresolution colorand texture features.” Ph.D. thesis Dept. Elect. Eng., Kyungpook Na-tional Univ., Daegu, Korea, 2005.

[15] J. P. P.S. Hiremath, "Content based image retrieval using color, texture and shape features, pp. 780784.” in Proceedings of the 15th International Conference on Advanced Computing and Communica-tions,Guwahati, India,, 2007.

[16] T. S. B.S. Manjunath, P. Salembier, "Introduction to mpeg-7: Multimedia content description interface,,” Wiley, Chichester, 2002.

[17] J. A. Q. Iqbal, "A system for content-based retrieval in digital image libraries," in International Conference on Control Automation,Robotics and Vision (ICARCV), 2002.

[18] A. S. N.Singha, K. Singhb, "A novel approach for content based image retrieval,” Procedia Technology, vol. 4, p. 245 250, 2012.

[19] Y. Y. Y. Rao, P. Mundur, "Fuzzy svm ensembles for relevance feedback in image retrieval,” LNCS, vol. 4071, p. 350359., 2006.

[20] X. L. X. W. D.C. Tao, X. Tang, "Asymmetric bagging and random subspace for svm-based relevance feedback in image retrieval," IEEE
Transactions on Pattern Analysis and Machine Vision, vol. 28 (7), p.1088-1099, 2006.

[21] P. G. U. Jayaraman, S.Prakash, "An efficient color and texture based iris image retrieval technique,” Expert Systems with Applications, vol. 39,p. 4915-4926, 2012.

[22] R. B. A. B. Gonde, R.P. Maheshwari, "Modified curvelet transform with vocabulary tree for content based image retrieval,” Digital Signal Processing, 2012.

[23] M. ElAlami, "A novel image retrieval model based on the most relevant features," Knowledge-Based Systems, vol. 24, p. 23-32, 2011.

[24] b. R. d. S. T. O. A.B. enatti a, Eduardo Valle a, "Comparative study of global color and texture descriptors for web image retrieval," J.VisCommun. Image R, vol. 23, p. 359-380, 2012.

[25] L. L. Z. F. J. Yuea, Zhenbo Li, "Content-based image retrieval using color and texture fused features," Mathematical and Computer Modelling, vol. 54, p. 1121-1127, 2011.

[26] E. K. H.N.pour, "Concept learning by fuzzy k-nn classification and relevance feedback for efficient image retrieval," Expert Systems with Applications, vol. 36, p. 5948-5954, 2009.

[27] S. S. E.Rashedi, H.Nezamabadi-pour, "A simultaneous feature adaptation and feature selection method 3 for content-based image retrieval systems,” Knowledge-Based Systems, 2012.

[28] H. Y. X.-Y. Wang, Y.J. Yu a, "An effective image retrieval scheme usingcolor, texture and shape features" Computer Standards \& Interfaces, vol. 33, p. 59-68, 2011.

[29] S. K. K. S. B. Park, J.W. Lee, "Content-based image classification using a neural network," Pattern Recognition Letters, vol. 25, p. 287-300, 2004.

[30] L. Y. G.H. Liu, Z.Y.Li, "Image retrieval based on microstructuredescriptor,” Pattern Recognition, vol. 44, p. 2123-2133,2011.

[31] M. K. K. M. Banerjee, "Edge based features for content based image retrieval,” Pattern Recognition, vol. 36, p. 2649-2661, 2003.

[32] E. R. A.M. Gonzlez-Quevedo, "Blobworld: A system for region-based image indexing retrieval," Proceeding of the 3rd International Conference on Visual Information Systems, p. 509-516, 1999.

[33] M. R. C. Veltkamp, "A survey of content-based image retrieval systems," Department of Computing Science, Utrecht University Utrecht,The Netherlands, pp. 47-101, 2002.

[34] G. S. B. Z. N. Jhanwar, S. Chaudhuri, "Content based image retrieval using motif co-occurrence matrix,," Image Vis. Comput, vol. 22, p.1211-1220., 2004.

[35] A. del Bimbo, "Visual information retrieval," Morgan Kaufmann Publishers, 1999.

[36] A. V. K.N. Plataniotis, “,color image processing and applications,”Springer, Berlin, 2000.

[37] B. D. P.L. Stanchev, D. Green Jr., "High level color similarity retrieval,,”Int. J. Inf. Theories Appl., vol. 10 (3), p. 363369, (2003).

[38] B. D. P.L. Stanchev, D. Green Jr., "High level color similarity retrieval,,”Int. J. Inf. Theories Appl., vol. 10 (3), p. 363-369, (2003).

[39] M. S. V. Mezaris, I. Kompatsiaris, "An ontology approach to object based image retrieval," Proceedings of the ICIP, vol. II, p. 511$514,2003$.

[40] B. Manjunath, "Color and texture descriptors," IEEE Trans CSVT, vol.11 (6), p. 703-715, 2001.

[41] S.-N. Y. C.-Y. Chiu, H.-C. Lin, "Texture retrieval with linguistic descriptors," IEEE Pacific Rim Conference on Multimedia, p. 308$315,2001$.

[42] K. Plataniotis, "Adaptive fuzzy systems for multichannel signal processing,” Proc. IEEE, vol. 87 (9), p. 1601-1622., 1999.

[43] R. Lukac, "Vector filtering for color imaging,," IEEE Signal Process.Mag, p. 74-86, 2005.

[44] C. W. C.M. Pun, "Fast and robust, color feature extraction for contentbased image retrieval,.” Int. J. Adv. Comput. Technol., vol. 3(6), (2011).

[45] D. B. M.J. Swain, “Color indexing,” International Journal of Computer Vision, vol. 7 (1), p. 11-32., 1991. 
[46] M. O. M.A. Stricker, "Similarity of color images," SPIE Storage and Retrieval for Image and Video Databases III, vol. 2420, p. 381392.,1995.

[47] A. F. R.O. Stehling, M.A. Nascimento, "Cell histograms versus color histograms for image representation and retrieval," Knowledge andinformation Systems, vol. 5 (3), p. 315-336, 2003.

[48] Y. C. C.Horng Lin, R.Tai Chen, "A smart content-based image retrieval system based on color and texture feature," Image and Vision Computing, vol. 27, p. 658-665, 2009.

[49] A. F. S. R. O. Stehling, M.A. Nascimento, "An adaptive and efficient clustering-based approach for content-based image retrieval in image databases,” International Database Engineering and Applications, p.356365, 2001.

[50] B. M. W.Y. Ma, "Netra: a toolbox for navigating large image databases.” Proceedings of the IEEE International Conference on Image Processing, p. 568-571, 1997.

[51] S. G. B.G. Prasad, K.K. Biswas, "Region-based image retrieval using integrated color, shape, and location index," Computer Vision and Image Understanding, vol. 94, p. 193233, 004.

[52] H. L. V. M. D. Levine, "Finding a small number of regions in an image using low-level features," Pattern Recognition, vol. 35, p. 23232339,2002.

[53] G. F. E.J. Pauwels, "Finding salient regions in images," J. Comput. Vision Image Understand, vol. 75, p. 73-85, 1999.

[54] R. D. A.K. Jain, “Algorithms for clustering data," Prentice-Hall, Inc, Englewood Cliffs, 1988.

[55] S. K. K. S. B. Park, J.W. Lee, "Content-based image classification using a neural network," Pattern Recognition Letters, vol. 25, p. 287-300, 2004.

[56] X. Y. Songhe, F. De Xu, “Attention-driven salient edge(s) and region(s) extraction with application to cbir,," Signal Processing, vol. 90, p. 115,2010 .

[57] D. C. G. Taubin, "Recognition and positioning of rigid objects using algebraic moment invariants," PIE Conference on Geometric Methodsin Computer Vision,, vol. 1570, p. 175-186., 1991.

[58] R. C. A.Mach, C.-H. Teh, "On image analysis by the methods of moments,” IEEE Trans. Pattern, vol. 10 (4), p. 496-513, 1988.

[59] G. L. D. Zhang, "Shape-based image retrieval using generic fourier descriptor," Signal Processing:Image Communication, vol. 17, p. 825848,2002 .

[60] G. S. B. Z. A.Hafiane, S. Chaudhurib, "Region-based cbir in gis with local space filling curves to spatial representation," Pattern Recognition Letters, vol. 27, p. 259-267, 2006.

[61] D. Z. G. L. Y. Liu, "Region-based image retrieval with high-level semantics using decision tree learning," Pattern Recognition, vol. 41,p. 2554- 2570, 2008.
[62] E. K. H. N.Pour, "Image retrieval using histograms of uni-colorband bicolor blocks and directional changes in intensity gradient," PatternRecognition Letters, vol. 25 (14), p. 1547- 1557, 2004.

[63] B. S. M. Wei-Ying Ma, "Netra: A toolbox for navigating large image databases,” Multimedia Systems, vol. 7(3), p. 184198, 1999.

[64] W. N. et al, "Querying images by content, using color, texture, and shape," in SPIE Conference on Storage and Retrieval for Image and Video Database, April 1993.

[65] T. H. , X.S. Zhou, "Relevance feedback in image retrieval: a comprehensive review,” Multimedia System, vol. 8 (6), p. 536-544., 2003.

[66] H.-Y. X.Y. Wang n, B.BeiZhang, "Active svm-based relevance feedback using multiple classifiers ensemble and features reweighting," Engineering Applications of Artificial Intelligence, vol. 26, p. 368-381, 2013.

[67] H.-Y. Y. Xiang-Yang Wang, Jing-Wei Chen, "A new integrated svm classifiers for relevance feedback content-based image retrieval using em parameter estimation,” Applied Soft Computing, vol. 11, p. 27872804,2011

[68] M. C. S.-C. C. C. Zhang, X. Chen, "A multiple instance learning approach for content based image retrieval using one-class support vector machine,” Proceedings of the IEEE International Conference on Multimedia and Expo, p. 1142-1145, 2005.

[69] T. H. Y. Rui, "Relevance feedback: A power tool for interactive image retrieval," IEEE Trans. on Circuits and Video Tech, 1998.

[70] V. Vapnik, "The nature of statistical learning theory," SpringerVerlag,1995.

[71] S. S. A. G. A. W. M. Smeulders, M. Worring and R. Jain, "Contentbased image retrieval at the end of the early years,,” IEEE Trans. Pattern Analysis and Machine Intelligence, vol. 22(12), pp. 1349-1380,2000.

[72] J. N. T .V .Ashwin and S. Ghosal, "Improving image retrieval performance with negative relevance feedback,,” Proc. IEEE Conf. on Acoustics, Speech, and Signal Processing (ICASSP'01), Utah, USA,2001.

[73] Q. T. P . Hong and V. a. T .S. Huang, "incorporate support vector machines to content-based image retrieval with relevant feedback, ,’Proc. IEEE Int. Conf. on Image Processing (ICIP’00), 2000

[74] S.Tong and E. Chang, ", support vector machine active learning for image retrieval,,” Proc. ACMMultimedia (MM’01), pp. 107-118., 2001.

[75] I. S. D. Stan, "Mapping low-level image features to semantic concepts,"in Proceedings of the SPIE: Storage and Retrieval for Media Databases, 2001.

[76] S H Shirazi, A I Umar, N UlHaq, S Naz, M I Razzak "Curvelet based Method for Red Blood Cell Image Segmentation” International WorkConference on Bioinformatics and Biomedical Engineering,2015.

[77] M I Razzak, B AlHaqbani "Automatic Detection of Malarial Parasite in Microscopic Blood Images” Journal of Medical Imaging \& Health Informatics, Volume 5, Issue 2, 2015. 